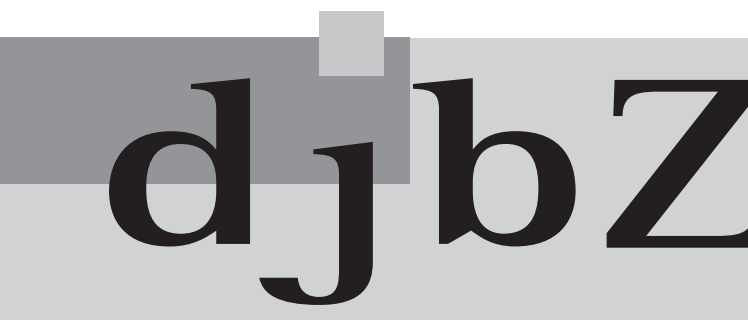

Zeitschrift des Deutschen Juristinnenbundes

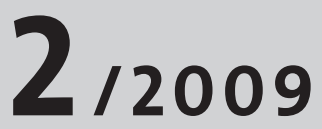

12. Jahrgang, Seite $51-100$

Herausgeber: Deutscher Juristinnenbund e. V.

Präsidium: Jutta Wagner, Rechtsanwältin und Notarin, Fachanwältin für Familienrecht, Berlin (Präsidentin); Renate Maltry, Rechtsanwältin, Fachanwältin für Familienrecht und Erbrecht, München; Ramona Pisal, Vorsitzende Richterin am Oberlandesgericht, Brandenburg a.d.H. (Vizepräsidentinnen); Dagmar Brinkmann, Regierungsdirektorin, Frankfurt a.M. (Schatzmeisterin); Schriftleitung: Anke Gimbal, Rechtsassessorin, Geschäftsführerin Deutscher Juristinnenbund, Berlin.

\title{
Vergewaltigung/Vergewaltigung in der Ehe
}

\section{- Anregungen aus der Rechtspraxis verschiedener europäischer Länder -*}

Sexualverbrechen stellen ein Phänomen von erheblicher Bedeutung dar. Von der UNO wird geschätzt, dass weltweit jede fünfte Frau mindestens einmal in ihrem Leben Opfer von Vergewaltigung oder sexueller Nötigung wird ${ }^{1}$. In Deutschland hatte eine breit angelegte Studie mit über 10.000 repräsentativ ausgewählten Frauen zwischen 16 und 85 Jahren zum Ergebnis, dass 13 Prozent der befragten Frauen - somit fast jede siebte - sexualisierte Gewalt erlebt haben; davon gaben sechs Prozent an, vergewaltigt worden zu sein, vier Prozent berichteten von einer versuchten Vergewaltigung, drei Prozent vom Zwang zu anderen sexuellen Praktiken. Sieben Prozent aller Frauen, die aktuell oder früher in einer Paarbeziehung leb(t)en, berichteten von sexualisierter Gewalt durch den (Ehe)Partner. ${ }^{2}$

Der Studie lässt sich weiter entnehmen, dass sexualisierte Gewalt zum großen Teil durch Partner, Expartner oder sonstige Familienangehörige ausgeübt wird ${ }^{3}$, wobei überwiegender Tatort die eigene Wohnung ist ${ }^{4}$. Aus einer sekundäranalytischen Auswertung der Untersuchung geht weiter hervor, dass sexualisierte Gewalt durch den Partner meist nicht als einmaliges Einzelereignis, sondern in der Regel als mehrmaliges Ereignis stattfindet ${ }^{5}$ und außerdem fast ausschließlich im Kontext von mehrmaliger, sehr schwerer körperlicher und erhöhter psychischer Gewalt auftritt ${ }^{6}$, folglich in besonderem Maße traumatisierende Folgen hat. Es liegen außerdem Erkenntnisse dahingehend vor, dass sexualisierte Gewalt als wichtiger Risikofaktor bei Tötungsdelikten zum Nachteil der Intimpartnerin angesehen werden muss ${ }^{7}$.
Es ist davon auszugehen, dass das Erleben von sexualisierter Gewalt in der Regel nicht nur langfristige soziale und psychosoziale Folgen für die Betroffenen nach sich zieht (z.B. Trennung, Scheidung, Wohnungswechsel, Aufnahme einer Therapie etc. $)^{8}$, sondern auch in erheblichem Maße die Gesundheit

Vortrag gehalten am 23.3.2009 in Paris als Vertreterin von EWLA (European Women Lawyers' Association) vor dem Komitee für die Gleichstellung von Mann und Frau der Parlamentarischen Versammlung des Europarats (PACE).

1 United Nations Secretary-General's Campaign „UNiTE”, Factsheet: How widespread is violence against women? http://endviolence.un.org/factsheets.shtml.

2 Studie, Lebenssituation, Sicherheit und Gesundheit von Frauen in Deutschland“, Repräsentative Untersuchung, Prof. Dr. Ursula Müller, Dr. Monika Schröttle, im Auftrag des Bundesministeriums für Familie, Senioren, Frauen und Jugend, Oktober 2004, Kurzfassung S. 7.

$359,4 \%$, Studie ,Lebenssituation ..." (Fn. 1) S. 12.

$469 \%$, Studie „Lebenssituation ...“ (Fn. 1) S. 12.

5 "Gewalt gegen Frauen in Paarbeziehungen“, eine sekundäranalytische Auswertung zur Differenzierung von Schweregraden, Mustern, Risikofaktoren und Unterstützung nach erlebter Gewalt (Kurzfassung), im Auftrag des Bundesministeriums für Familie, Senioren, Frauen und Jugend, Dr. Monika Schröttle, November 2008 , S. 15.

6 Ebd., S. 15 und 20; dies wird gestützt durch die Beobachtung der Praxis, dass im Rahmen von Ermittlungen nach Anzeigen wegen körperlicher Gewalt nicht selten Sexualverbrechen erstmals offenbar werden, die zuvor nicht angezeigt wurden.

7 "If only we'd known“ - an explanatory study of seven intimate partner homicides, Linda Regan, Liz Kelly, Anne Morris, Rebecca Dibb, CWASU (Child and Woman Abuse Studies Unit) London Metropolitan University, S. 18 (m.w.N.).

8 Wobei dies nicht nur bei (sexualisierter) Gewalt durch den Partner, sondern auch nach Gewalterleben durch fremde Täter gilt: Studie „Lebenssituation” (Fn. 1) S. 15.
Sabine

\section{Kräuter-Stockton}

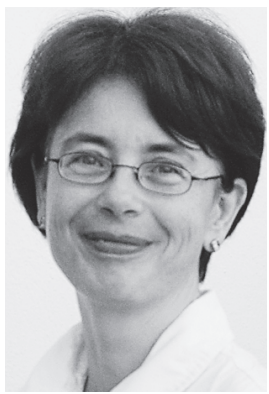

Vorsitzende des Landesverbands Saarland im djb, Mitglied der Kommission Gewalt gegen Frauen und Kinder des djb, Staatsanwältin, Saarbrücken 
der Opfer beeinträchtigt: bei Frauen, die sexualisierte Gewalt erlebt haben, ist ein erhöhter Alkohol- und Medikamentenkonsum sowie ein viel höherer Tabakkonsum zu beobachten, sie sind durch gesundheitliche Beschwerden verschiedenster Art in weit stärkerem Maße belastet als Frauen, die nie Opfer von sexueller Nötigung oder Gewalt waren $^{9}$.

\section{Vergewaltigung}

\section{Tatbestandsmerkmal: ,gegen den Willen“ oder „mit Gewalt“}

Europaweit werden je nach Rechtsordnung unterschiedliche materiell-rechtliche Definitionen für den Tatbestand der sexuellen Nötigung oder Vergewaltigung gebraucht, wobei Länder, die ihr Strafverfahren nach dem Untersuchungsgrundsatz füh-

\section{Wenn - wie in einer Reihe europäischer Länder - eine Vergewaltigung durch den Vollzug des Geschlechts- verkehrs ohne Einverständnis des Opfers definiert wird} (ohne zusätzliches Erfordernis der Gewaltanwendung), können strafwürdige Fälle vor Gericht gebracht werden, die anderenfalls unbestraft bleiben.

ren, in der Regel die Anwendung von Gewalt oder qualifizierter Drohung zur Überwindung des entgegenstehenden Willens des Opfers verlangen, wogegen die Länder mit kontradiktorischem Rechtssystem den Fokus eher auf den entgegenstehenden Willen oder das fehlende Einverständnis des Opfers legen ${ }^{10}$. Wenn auch bei beiden Varianten gleichermaßen ein Großteil der Probleme auf dem Weg zu einer Verurteilung des Täters in Schwierigkeiten mit der Beweisführung vor Gericht gesehen wird ${ }^{11}$, so ist doch andererseits festzustellen, dass in den Ländern, in denen - wie z.B. in Deutschland - die Anwendung von Gewalt oder einer qualifizierten Bedrohung des Opfers zur Überwindung dessen Widerstands verlangt wird, eine Anzahl strafwürdiger Fälle bereits zu Beginn des Ermittlungsverfahrens ausgeschieden werden: Die Frau, die aufgrund der ungleichen Kräfteverhältnisse ihren möglichen Widerstand von vornherein als aussichtslos einschätzt, die Angst hat, mit aktivem Widerstand den Täter so zu provozieren, dass er ihr möglicherweise noch Schlimmeres antun würde, die mit tränenüberströmtem Gesicht ruft, dass sie „das“ nicht will und sich steif macht wie ein Brett, die aber keinen aktiven Widerstand leistet, den der Täter zur Tatausführung hätte brechen müssen, diese Frau fühlt sich vergewaltigt. Moralisch gesehen hat sie damit auch Recht, sie wird aber auf ihre Anzeige hin in der Regel einen Einstellungsbescheid erhalten, in dem bedauert wird, dass angesichts dieser Umstände - keine Gewaltanwendung - eine Anklage ausscheidet. Diese Fälle gelangen überhaupt nicht vor Gericht. Dies ist nicht sachgerecht.

Wenn dagegen, wie in einer Reihe europäischer Länder ${ }^{12}$, eine Vergewaltigung durch den Vollzug des Geschlechtsverkehrs ohne Einverständnis des Opfers definiert wird (ohne zusätzliches Erfordernis der Gewaltanwendung), können strafwürdige Fälle vor Gericht gebracht werden, die anderenfalls unbestraft bleiben. Die hiergegen gern polemisch eingewendete Besorgnis, ein Mann, der nicht Gefahr laufen wolle, als Vergewaltiger bestraft zu werden, müsse dann vor dem Geschlechtsverkehr ein schriftliches Einverständnis der Frau einholen, geht ersichtlich an der Sache vorbei: selbstverständlich würde auf der Ebene des Vorsatzes geprüft werden müssen, ob dem Täter aus seiner Sicht klar erkennbar war, dass er gegen den entgegenstehenden Willen der Frau handelt. Nur dann würde er sich strafrechtlichen Vorwürfen ausgesetzt sehen.

Von den europaweit zwei unterschiedlichen Varianten für die Erfordernisse an eine „Vergewaltigung/sexuelle Nötigung “ im Rechtssinne ist diejenige vorzugswürdig, die lediglich ein beweisbares fehlendes Einverständnis des Opfers mit den sexuellen Handlungen verlangt.

\section{Gründe für niedrige Anzeigequote}

Es ist bekannt, dass Sexualstraftaten noch seltener angezeigt werden als häusliche Gewalt ${ }^{13}$. Gründe hierfür liegen in unter anderem einer hohen Schamschwelle sowie insbesondere in der Angst der Opfer, dass „niemand glauben wird“, was geschehen ist, Angst davor, von der Polizei und vor Gericht durch die Mühle gedreht zu werden und das Innerste nach außen kehren zu müssen, mit einem ungewissen Ausgang.

\section{„Kultur des Unglaubens“}

Tatsächlich kommt es immer wieder - trotz Sensibilisierung und Fortbildungen - in der Praxis vor, dass den Anzeigerinnen von vornherein Unglauben und Misstrauen entgegenschlagen. Bei Polizei und Justiz herrscht oft das Vorurteil, dass Anzeigen

9 Studie „Lebenssituation, Sicherheit und Gesundheit von Frauen in Deutschland", Langfassung, S. 152 f.

10 „Rape: the forgotten issue”, Prof. Liz Kelly u. Linda Regan, Child and Woman Abuse Studies, London, S. 26. 11 Ebd.

12 Z.B. Großbritannien: Section 1, 74 des Sexual Offences Act von 2003.

13 „Rape: the forgotten issue?” (s. Fn. 10), S. 2. 
wegen Sexualdelikten häufiger vorgetäuscht würden als Anzeigen wegen anderer Delikte ${ }^{14}$. Wenn parallel ein familienrechtliches Gerichtsverfahren läuft, in dem zum Beispiel um das Umgangsrecht mit den Kindern gestritten wird, entsteht oft die Besorgnis, die Strafgerichtsbarkeit werde „instrumentalisiert“, der Vorwurf sei erfunden, um einen günstigen Ausgang des Familienverfahrens zu erreichen. Wenn man Praktiker fragt, zeigt sich außerdem manchmal eine übertriebene professionelle Skepsis $^{15}$ hinsichtlich der Häufigkeit von Falschanzeigen: Schätzungen von professionellen Ermittlern, wie viele Anzeigen wegen Vergewaltigung ihrer Meinung nach vorgetäuscht sind, liegen oft erheblich über deren tatsächlichem Anteil ${ }^{16}$. Dies könnte daran liegen, dass aus der Enttäuschung über eine einmal erlebte Falschanzeige im Rückblick die Gesamtanzahl von Falschanzeigen völlig überschätzt wird. Auch kommt es oft vor, dass eine zweite, widerrufende Aussage ungeprüft für wahr gehalten wird. Die zweite Aussage einer Anzeigerin ist aber nicht unbedingt zutreffend, auch wenn sie sich damit selbst einer Straftat bezichtigt. Es wäre durchaus nachvollziehbar, wenn ein Vergewaltigungsopfer einen Rückzieher macht, nachdem es gemerkt hat, was es im weiteren Lauf des Verfahrens zu erwarten hat.

Eine Erforschung dieses Phänomens mit einer breit angelegten wissenschaftlichen Untersuchung ist meines Wissens noch nicht erfolgt.

Eine wissenschaftliche Studie zum tatsächlichen Ausmaß von Vortäuschungen und zur diesbezüglichen Fehleinschätzung durch Polizei und Justiz könnte, verbunden mit breiter Öffentlichkeitsarbeit, Vorurteilen bei Professionellen entgegenwirken. Dies würde sich positiv auf die Qualität der Arbeit und längerfristig auf die Anzeigequote bei Vergewaltigungen auswirken.

\section{Erörterung der sexuellen Vorgeschichte/des sexuel- len Vorlebens des Opfers}

Ein anderer wichtiger Grund für fehlende Anzeigebereitschaft im Fall von Sexualdelikten liegt in der oft auch zutreffenden Erwartung der Opfer, dass sie vor Gericht im Rahmen einer intensiven Befragung zu ihrem sexuellen Vorleben ihr Intimleben entblößen müssen und sich einer entwürdigenden Behandlung ausgesetzt sehen. Tatsächlich nimmt in der Praxis nicht selten die Befragung zu dem von der angeklagten Tat unabhängigen sexuellen Vorleben der Geschädigten ein Ausmaß an, das es verständlich macht, wenn aus Angst vor dieser Prozedur gar nicht erst Anzeige erstattet wird. Es lässt sich oft der Eindruck nicht von der Hand weisen, dass mit solchen Fragen zum Intimleben versucht wird, das Opfer in den Schmutz zu ziehen, es als Person unglaubhaft zu machen und es zu zermürben, um die Wahrheit zu verdunkeln. Im Hinblick auf dieses Problem wurde in Großbritannien beispielsweise bereits 1999 eine Regelung eingeführt, wonach Fragen an das Opfer einer Sexualstraftat $\mathrm{zu}$ seinem sexuellen Vorleben nur unter bestimmten, eng begrenzten Voraussetzungen zulässig sind ${ }^{17}$. Wenn auch berichtet wird, dass diese Regelung zuweilen in der Praxis nicht befolgt und weiterhin in gewohnter Weise vor Gericht verfahren wird $^{18}$, so ist zu erwarten, dass mit einer neuen Generation von Juristen im Lauf der Jahre die Regelung durch die Praxis zunehmend befolgt werden wird.

\section{Schätzungen von professionellen Ermittlern, wie viele Anzeigen wegen Vergewaltigung ihrer Meinung nach vorgetäuscht sind, liegen oft erheblich über deren tat- sächlichem Anteil.}

Die Einführung gesetzlicher Regelungen, die eine Erörterung der sexuellen Vorgeschichte des Opfers vor Gericht nur unter bestimmten Voraussetzungen erlauben, kann auf lange Sicht die Anzeigebereitschaft der Opfer von Sexualdelikten stärken.

\section{Anonyme Spurensicherung vor Anzeigeerstattung}

Bei Opfern von Sexualdelikten besteht häufig erst nach Monaten oder Jahren der psychosozialen Unterstützung und Stabilisierung die Kraft und die Bereitschaft, eine Anzeige zu erstatten. Bis dahin sind in der Regel rechtsmedizinisch zu sichernde Spuren, die unmittelbar nach der Straftat noch zur Überführung des Täters hätten herangezogen werden können, vernichtet. Üblicherweise ist eine medizinische Untersuchung, die nicht der Behandlung, sondern der Spurensicherung dient, nur bei gleichzeitiger Anzeigeerstattung bei den Strafverfolgungsbehörden möglich, die dann auch die Kosten hierfür im Rahmen des Strafverfahrens übernehmen. Verschiedentlich wird daher in (rechts)medizinischen

14 Obwohl es gerade umgekehrt zu sein scheint: so zumindest Hinweise in „Rape ..." (Fn. 8) S. 31.

15 Elsner, Erich; Steffen, Wiebke: Vergewaltigung und sexuelle Nötigung in Bayern, im Auftrag des Bayerischen Staatsministeriums des Inneren, München 2005 S. 182.

16 „Rape: the forgotten issue?” (s. Fn. 8) S. 33.

17 Youth Justice and Criminal Evidence Act 1999, Chapter III.

18 British Home Office Online Report 20/06 „Sect. 41: an evaluation of new legislation limiting sexual history evidence in rape trials" by Liz Kelly u.a. http://www.homeoffice.gov.uk/rds/pdfso6/rdsolr20o6.pdf. 
Instituten ${ }^{19}$ eine von Anzeigeerstattung unabhängige Spurensicherung an Opfern von Sexualstraftaten ermöglicht. Die aufgefundenen Beweismittel werden dann bis zum Zeitpunkt der Anzeigeerstattung sachgerecht aufbewahrt ${ }^{20}$ und können zu gegebener Zeit als objektive Beweismittel in angemessener Weise zur Wahrheitsfindung beitragen.

Anzeigeunabhängige rechtsmedizinische Spurensicherung ermöglicht, objektive Beweismittel auch noch längere Zeit nach der Tat in ein Verfahren einzubringen.

\section{Vergewaltigung in der Ehe}

\section{Strafmilderungsgrund}

Ende der 1990er Jahre wurden in fast allen Ländern Europas Sexualverbrechen, auch wenn sie innerhalb der Ehe begangen waren, unter Strafe gestellt, während nach alter Rechtslage noch weitgehend die Vergewaltigung in der Ehe straflos war. Überwiegend erfolgte die Änderung nicht mittels Schaffung eines eigenen Straftatbestands „Vergewaltigung in der Ehe“, sondern durch Streichung

\section{Die Rechtspraxis behandelt teilweise eine Ehe zwischen Täter und Opfer als Grund für eine Strafmilderung. Das ist rechtssoziologisch wie kriminologisch verfehlt und muss als Relikt aus früheren Jahrhunderten gebrandmarkt werden.}

der früher üblichen Ausnahmeregelung für Ehemänner ${ }^{21}$. Die Abschaffung dieses Ausnahmetatbestands ist ein Signal für das Ende der in Europa in früheren Zeiten etablierten patriarchalen Herrschaftsverhältnisse, die es Ehemännern erlaubten, ihren Frauen im Ehebett ihren Willen aufzuzwingen und somit Ehefrauen rechtlich auf die Stufe einer Sache stellten (Vergewaltigung der eigenen Ehefrau war straflos, so wie die Beschädigung der eigenen Sache). Das Gewaltmonopol des Staates, das mit unserer Vorstellung von Rechtsstaatlichkeit eng verknüpft ist, verlangt, dass es in unseren Gesellschaften keinen straffreien Raum geben darf. Hinzu kommt die Erkenntnis, dass ein Sexualverbrechen, erlitten durch den eigenen Ehemann oder Partner in der vermeintlichen Sicherheit der eigenen Wohnung, das Opfer mindestens ebenso schwer traumatisiert wie eine durch einen Fremden verübte Tat, zumal Untersuchungen zeigen, dass durch den Partner begangene sexualisierte Gewalt in der Regel von erhöhter physischer und psychischer Gewalt begleitet ist ${ }^{22}$.
Nach heutiger europaweiter Rechtslage besteht zwar kein Zweifel mehr an der generellen Strafbarkeit der Vergewaltigung in der Ehe. Dennoch wird in der Rechtspraxis häufig die Tatsache, dass der Täter mit dem Opfer verheiratet ist (oder war) oder mit ihm in einer Lebensgemeinschaft lebt(e), als Grund für eine Strafmilderung angesehen ${ }^{23}$ - was rechtssoziologisch wie auch kriminologisch verfehlt ist und als Relikt aus früheren Jahrhunderten gebrandmarkt werden muss. Sinn und Zweck einer Strafe werden konterkariert, wenn eine bestehende oder bestandene Ehe/Lebensgemeinschaft als Grund für eine Strafmilderung gewertet wird und damit die besonders schwere Beeinträchtigung und Demütigung des Opfers einer Vergewaltigung gerade durch den (Ehe)Partner bagatellisiert werden.

Eine bestehende oder frühere Ehe/Lebensgemeinschaft zwischen Täter und Opfer darf nicht als Strafmilderungsgrund bei Sexualstraftaten gewertet werden.

\section{Offizialprinzip}

Angesichts der Schwere des Tatvorwurfs, der Folgen für das Opfer und der menschenverachtenden Gesinnung, die der Täter durch die Tatbegehung offenbart, sollte es selbstverständlich sein, dass auch dann, wenn die Sexualstraftat innerhalb einer Ehe stattfand, der Staat von Amts wegen Strafverfolgung betreibt und die Strafverfolgung nicht von einer Initiative oder einem Strafantrag des Opfers abhängt ${ }^{24}$.

Das Offizialprinzip muss für alle Fälle der Sexualstraftaten gelten, unabhängig von einer Ehe.

\section{Zeugnisverweigerungsrecht}

Die Rechtsordnungen Europas sehen praktisch alle ein Zeugnisverweigerungsrecht der Ehefrau im Verfahren gegen ihren Ehemann vor. Da bei sexueller Gewalt wie auch bei sonstiger Partnerschaftsgewalt ein stark ambivalentes Verhältnis zwischen Täter und Opfer die Regel ist und das Aussagever-

19 Unikliniken Hamburg-Eppendorf, Deutsches Ärzteblatt 27.12.1999 s. http://www.aerzteblatt.de/v4/archiv/artikel.asp? src $=$ heft\&id=20579 und Münster, http: $/ /$ www. klinikum.uni-muenster.de/shownews. php?id=1694.

20 Siehe auch das Dortmunder Projekt „Anonymisierte Spuren- und Beweissicherung nach Vergewaltigung", Petra Hermes in aktuelle Informationen des djb-Deutscher Juristinnenbund 3/2007, S. 13 f.

21 So auch in Spanien Art. $178 \mathrm{ff}$. des Codigo Penal von 1995.

22 S. Fußnoten 5 und 6.

23 In England: Liz Kelly in newstatesman.com vom 12.3.2008; in Deutschland: z.B. Bundesgerichtshof BGH 2 StR 276/01 vom 26.8.2001.

24 So aber z.B. in Slowenien: Strafverfolgung wegen Vergewaltigung in der Ehe nur, wenn das Opfer Strafantrag stellt. 
halten des Opfers ebenso wie sein übriges Verhalten gegenüber dem Täter von Angst, Hoffnung auf Besserung, Scham und vielen anderen Einflüssen bestimmt ist, ist in der Praxis häufig zu beobachten, dass trotz Anzeigeerstattung und anfänglich hoher Aussagebereitschaft in den Monaten (oder Jahren) bis zur Hauptverhandlung vor Gericht die Aussagebereitschaft schwindet. Die geschädigten Frauen wissen, dass staatliche Stellen nur eine begrenzte Macht besitzen, um sie vor dem Täter zu schützen. Selbst wenn er längerfristig inhaftiert wird, wird er irgendwann aus dem Gefängnis entlassen und wird sich dann für die Aussage des Opfers vor Gericht zu rächen wissen. Auch die Hoffnung des Opfers, dass der Täter, der sich während des Laufs des Strafverfahrens zu beherrschen weiß, sein Verhalten künftig bessern und Ähnliches nicht wiederholen werde, kann zu Aussageverweigerung des Opfers vor Gericht führen. Je nach Rechtsordnung unterschiedliche Regelungen - in Deutschland steht neben der Ehefrau auch der Verlobten ${ }^{25}$, in Spanien auch der Lebensgefähr$\operatorname{tin}^{26}$ ein Zeugnisverweigerungsrecht $\mathrm{zu}$ - und Beweisverwertungsverbote zwingen bei späterer Aussageverweigerung der Geschädigten dann nicht selten zu einer Einstellung des Verfahrens oder zum Freispruch, obwohl alle Beteiligten - Juristen, Angeklagter, Opfer - wissen, dass die Tat stattgefunden hat.

In Kenntnis dieser Rechtslage greifen nicht selten Täter, die (sexualisierte) Gewalt gegenüber einer zeugnisverweigerungsberechtigten Person ausgeübt haben, zu massivem Druck, teilweise zur Anwendungen von Drohungen oder von erneuter Gewalt, um hierdurch eine Zeugnisverweigerung des Opfers vor Gericht und damit einen Freispruch zu erwirken.

Diesem Problem wird in manchen Rechtsordnungen ${ }^{27}$ dadurch begegnet, dass eine nach qualifizierter Belehrung und unter bestimmten Umständen vor der Hauptverhandlung abgegebene Aussage auch bei nachträglicher Zeugnisverweigerung im Prozess verwertet werden kann. Dies wird wohl eher selten als alleiniges Beweismittel für eine Verurteilung des Täters ausreichen, doch kann bei solcher Regelung in Verbindung mit weiteren Zeugenaussagen (etwa vom Hörensagen) oder mit ärztlichen Attesten eine Verurteilung erfolgen, ohne dass das Opfer Auge in Auge gegenüber dem Täter die ihn belastende Aussage machen und sich hinterher ihm gegenüber dafür verantworten muss. Das Bewusstsein, dass es sich dann nicht auf den Ausgang des Verfahrens auswirkt, ob die Geschädigte vor Gericht aussagt oder nicht, kann zu einer enormen Entlastung bei ihr führen. Es ist weiter zu erwarten, dass eine solche Regelung den zusätzlichen Druck des Täters auf das Opfer, um es zu Zeugnisverweigerung noch vor Gericht zu zwingen, entfallen lässt.

Im Übrigen ist es nicht sachgerecht, ein Zeugnisverweigerungsrecht an förmlich nicht geregelte und gerichtlich kaum überprüfbare Lebenssachverhalte wie ein Verlöbnis oder eine Lebensgemeinschaft ${ }^{28}$ zu knüpfen. Das „Verlöbnis“ in Deutschland ist bereits dann nicht auszuschließen, wenn die Behauptung eines formlosen Eheversprechens, d.h. gegensei- tige Erklärung der Verlobten, einander heiraten zu wollen, nicht zu widerlegen ist. In der Praxis ist zu beobachten, dass diese Rechtslage nicht selten einen Missbrauch geradezu provoziert: Im Extremfall „verlobt“ sich der Angeklagte mit der Hauptbelastungszeugin vor dem Gerichtssaal, worauf ihre frühere Aussage nicht mehr zu verwerten und der Angeklagte meist freizusprechen ist. Nach Rechtskraft des Urteils kann dann die „Entlobung“ wieder genauso formlos stattfinden die effektive Strafverfolgung und Wahrheitsfindung haben das Nachsehen. Das Zeugnisverweigerungsrecht für Verlob$\mathrm{te}^{29}$ und Lebensgefährten ist daher verfehlt - insbesondere wenn es um (sexuelle oder körperliche) Gewalt geht.

Eine Aufweichung der Regelungen hinsichtlich des Zeugnisverweigerungsrechts (sowohl betreffend Anknüpfungstatsachen wie Verlöbnis und Lebensgemeinschaft, als auch betreffend Beweisverwertungsverbote) kann die in Fällen von Vergewaltigung in der Ehe häufige Einwirkung des Täters auf die Hauptbelastungszeugin vor Gericht entschärfen und die Geschädigte entlasten.

\section{Parallele Problemlagen in Europa trotz unterschiedlicher Rechtsordnungen}

Aus Sicht der Rechtspraxis in verschiedenen europäischen Ländern haben sich mehrere Problempunkte um das Phänomen „Vergewaltigung/in der Ehe“ herauskristallisiert, die den Praktikerinnen unter den Nägeln brennen und - trotz jeweils unterschiedlicher Rechtsordnungen - erstaunlich viele Parallelen aufzeigen. Es bleibt abzuwarten, ob das zähe Ringen um Verbesserungen auf diesem Gebiet in den nächsten Jahrzehnten Früchte bringt.

$25 \S 52$ StPO; wobei keinerlei formelle Anforderungen an das Vorliegen eines Verlöbnisses gestellt werden, somit auch eine Überprüfung des tatsächlichen Bestehens eines Verlöbnisses praktisch nicht möglich ist.

26 Art. 416 der Ley de Enjuiciamiento Criminal in Verbindung mit dem Urteil des Tribunal Supremo 134/07 vom 22. Februar; siehe "Guia de criterios de actuación judicial frente a la violencia de género " des Consejo General del Poder Judicial (spanisches Kollegialorgan/Richterverwaltungsgremium im Justizministerium), zu finden unter: http://www.observatorioviolencia.org/documentos.php?id=188.

27 Z.B. in Slowenien, wo seit Kurzem eine nach qualifizierter Belehrung vor dem Untersuchungsrichter abgegebene Aussage eines Opfers vor Gericht auch dann verwertet werden kann, wenn das Opfer nachträglich im Prozess von seinem Zeugnisverweigerungsrecht Gebrauch macht.

28 S. Fn. 23 und 24.

29 So auch der in den Deutschen Bundestag eingebrachte Gesetzentwurf des Bundesrats 16/516 vom 02.02.2006 http://www.bundestag.de/aktuell/hib/2006/2006_043/04.html. 